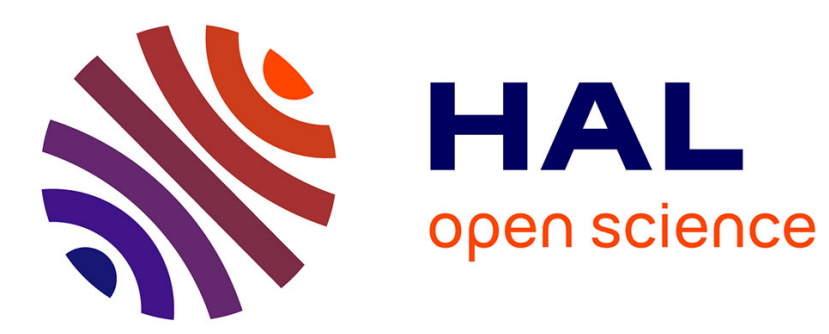

\title{
De la notion de détachement topical à celle de constituant thématique extrapropositionnel
}

\author{
Anne Lacheret, Jacques François
}

\section{To cite this version:}

Anne Lacheret, Jacques François. De la notion de détachement topical à celle de constituant thématique extrapropositionnel. Les cahiers de praxématique, 2003, Linguistique du détachement, 40, pp.167-198. 10.4000/praxematique.2730 . halshs-00364047

\section{HAL Id: halshs-00364047 https://shs.hal.science/halshs-00364047}

Submitted on 25 Feb 2009

HAL is a multi-disciplinary open access archive for the deposit and dissemination of scientific research documents, whether they are published or not. The documents may come from teaching and research institutions in France or abroad, or from public or private research centers.
L'archive ouverte pluridisciplinaire HAL, est destinée au dépôt et à la diffusion de documents scientifiques de niveau recherche, publiés ou non, émanant des établissements d'enseignement et de recherche français ou étrangers, des laboratoires publics ou privés. 
Anne Lacheret, Jacques François

Laboratoire CRISCO

Université de Caen

14032 Caen cedex

\{anne.lacheret, jacques.francois\}@crisco.unicaen.fr

De la notion de détachement topical à celle de constituant thématique extrapropositionnel

\section{Introduction}

Dans cet article, nous porterons toute notre attention sur un certain type de constructions détachées: les détachements topicaux en position frontale d'énoncé qui instancient une ou plusieurs entités référentielles au sujet desquelles la proposition qui suit apporte une information pertinente (ex. la langue française, elle est compliquée mais elle est drôle). Notre contribution est organisée de la façon suivante :

- Nous commençons par présenter la notion de détachement telle qu'elle a pu être appréhendée par les grammaires fonctionnelles et plus particulièrement par S. Dik et K. Lambrecht. A la suite de ces auteurs, nous défendons l'hypothèse que, en français parlé tout du moins, le détachement topical ne résulte pas d'une dislocation syntaxique, c'est-à-dire de la transformation d'une structure syntaxique première, naturellement liée. En d'autres termes, le topic 1 n'est pas extrait de la clause ${ }^{2}$, bien au contraire, la clause s'ajuste à lui. Ceci nous conduira à questionnner la notion même de détachement et à poser ses limites. Existe-t-il des contextes où la notion s'impose vraiment ? Quels sont ceux qui ne la convoquent pas explicitement? Dans ces derniers, quel concept lui substituer?

\footnotetext{
${ }^{1}$ Nous employons indifféremment les termes thème et topic.

2 Au sens de proposition ici.
} 
Telles seront nos questions ici. Au terme de cette réflexion, nous serons amenés à introduire la notion de constituant thématique extrapropositionnel (CTE), que nous appréhendons sous l'angle cognitif : le CTE est une unité perceptivement saillante, qui se détache comme une figure sur un fond dans le fil discursif, pour servir des fonctions pragmatiques précises.

- Nous exposons ensuite les marqueurs intonosyntaxiques susceptibles d'être mobilisés pour activer cette saillance. Sous l'angle syntaxique d'abord, en nous fondons sur des exemples de français parlé en situation de dialogue à bâtons rompus (émission les fous du roi, France Inter 2002, conversations familières), nous proposons un classement des détachements topicaux qui affine et prolonge les typologies déjà posées (voir notamment Lambrecht 2002). Du point de vue intonatif ensuite, nous proposons un modèle morphologique, hiérarchique de l'intonation (Rossi 1999, Lacheret 2002). Nous montrons comment une modélisation en termes de contours globaux peut conduire à l'émergence d'un paradigme d'intonèmes, l'actualisation de l'un ou l'autre de ces intonèmes sur le segment thématique pouvant lui conférer différents degrés de saillance.

- La dernière section est consacrée à l'interprétation fonctionnelle de ces constructions. Dans un premier temps, l'alignement des structures syntaxique et intonative, nous permet de préciser la notion de degré amorcée dans la section précédente et d'exploiter la redondance des marques sous un angle écologique (tel que peut l'exploiter un auditeur pour passer d'un percept brut à sa représentation cognitive). Nous explorons ensuite deux pistes pour préciser (i) les relations entre le type de construction intonosyntaxique produit et le degré d'accessibilité cognitive du constituant extraporpositionnel (actif, accessible, inactif); (ii) le rôle co-énonciatif de ces constructions. Dans cette dernière section, il s'agit de mener conjointement une réflexion sur les opérations cognitives élé- 
mentaires (ou principes d'activation) sous-jacentes aux constructions analysées (principe d'ancrage, de distance, de monstration).

2. Le détachement : définition du concept et présentation de ses marquages intonosyntaxiques

La notion de détachement topical est-elle fondée dès lors qu'on aborde le matériau oral ? Comment l'appréhender dans ce contexte? Quels en sont les marqueurs linguistiques? Telles sont les questions qui font l'objet de cette section.

\subsection{Une notion cognitive et non transformationnelle}

Commençons par évoquer la position de Simon Dik (1997), qui constitue l'aboutissement le mieux argumenté de la théorie de la perspective fonctionnelle de la phrase initiée par l'école de Prague dans les années 50 et développée ultérieurement par l'école fonctionnelle sytémique de M.A.K. Halliday (cf. Halliday \& Hasan 1976, Halliday 1985).

Promoteur du modèle néerlandais de grammaire fonctionnelle (1997, Section 17.2.3), S. Dik opère une distinction fondamentale entre constituants intra- et extrapropositionnels (clausal vs. extraclausal constituent). Dans la terminologie de la Functional Grammar, un constituant topical est désigné comme Topic s'il est intrapropositionnel et comme Theme s'il est extrapropositionnel. Du point de vue de la gestion de l'acte de communication, la notion même de constituant extrapropositionnel est préférable à celles de constituant "détaché", "disloqué" ou "extraposé" qui, par l'emploi d'un participe passé, introduisent explicitement ou suggèrent implicitement une opération ou transformation d'extraction d'un constituant de la proposition. S. Dik se place dans une toute autre perspective en proposant une reformulation de la maxime de pertinence de Grice 
(1975) destinée à définir les relations admissibles entre un Thème et une Proposition : "Pour qu'une paire quelconque d'un thème $T$ et d'une proposition $P$ fasse sens, il doit être pertinent de prononcer $P$ en considération de $T$ ". En d'autres termes, l'introduction d'un énoncé par un Thème (extrapropositionnel) vise à désigner une entité ou un ensemble d'entités par rapport auxquelles la proposition qui suit va apporter une information pertinente.

Dans son argumentation visant à rejeter l'expression “ constituant détaché ", Dik insiste sur les cas où la formulation d'une règle tranformationnelle est impossible, lesquels relèvent dans ses exemples exclusivement de l'anaphore associative, ex. As for Paris, the Eiffel Tower is really spectacular, mais doivent inclure également les cas de détachement à SN non lié (cf. Lambrecht 2002, ex. La mer / tu vois de l'eau). Evoquant la classe des langues à topic proéminent de Li \& Thompson (1976), il observe qu'à un degré plus ou moins marqué, toutes les langues font usage de ce type d'organisation ${ }^{3}$. Dik accorde une force illocutoire propre à certains constituants dotés d'une fonction discursive thématique, en particulier en cas de reprise partielle en écho de la partie focale d'une question de l'in-

3 Hagège (1978) évoque des cas d'évolution cyclique, où certaines langues procèdent en diachronie à la séparation du sujet et du topic puis à leur réunification. On sait que le français standard écrit ou oral soutenu ne sépare pas le sujet du topic en ce sens que le sujet représenté par un SN est un constituant topical intrapropositionnel (ex. Alors, ton ami t'a rendu ton livre?). Si l'on considère les contraintes d'ordre des SN sujet et objet direct comme un type de marquage, cette variante du français entre dans le type des LANGUES A MARQUAGE SUR LES MEMBRES (dependent marking languages). En revanche, le français de la conversation relâchée abonde de SN thématiques extrapropositionnels repris par un pronom clitique (ex. Alors ce livre, il te l'a rendu, ton copain ?), configuration caractéristique des LANGUES A MARQUAGE SUR LES TETES (head marking languages) dans le classement proposé par van Valin \& LaPolla (1997:23-25) et van Valin (2001:99-100). 
terlocuteur. L'énoncé est alors analysé comme la conjonction d'un constituant à valeur initiale de Focus dans le tour de parole précédent et requalifié en Thème, et d'une proposition liée (cf . infra 3.2, pour un point de vue intonatif) :

\begin{tabular}{l|ll} 
& My brother $\uparrow$ & $\begin{array}{l}\text { I haven't seen him } \\
\text { for years } \downarrow\end{array}$ \\
\hline $\begin{array}{l}\text { Statut } \\
\text { fonctionnel }\end{array}$ & THEME & PROPOSITION \\
Force illocutoire: & QUESTION & DECLARATION
\end{tabular}

Dik évoque aussi les phénomènes d'hésitation avant la formulation de la proposition (ex. As for the students, well, let me see) pour énoncer sa thèse centrale : "Le thème n'est pas extrait de la proposition; au contraire la proposition est ajustée au thème". (1997: 393). L'argument est effectivement pertinent, mais il demanderait à être articulé sur les expérimentations psycholinguistiques menées sur la planification du discours entre 'conceptualisation' et 'formulation' (Pour une revue, voir Fayol 2002).

Dans cette mouvance, Lambrecht $(1994,2002)$ nous intéresse particulièrement étant donné l'application de ses travaux au français parlé. Selon l'auteur, l'énoncé est pragmatiquement structuré autour de deux éléments centraux : le topic, ou élément donné, dont on dit quelque chose, accessible par le contexte discursif ou situationnel et le focus, qui correspond à l'information nouvelle. Parmi les différents types énonciatifs qui peuvent actualiser la structure informationnelle, émergent en premier lieu les constructions à fous prédicatif, organisées autour d'un détachement topical gauche (L-TOP) ou droit (R-TOP) dont la fonction discursive de base est d'introduire un référent qui n'a pas encore été ratifié dans le discours (L-TOP) ou de maintenir un référent topical déjà ratifié (R-TOP) 
(Lambrecht 1998). C'est le premier qui nous intéresse ici. Selon Lambrecht, ce type de construction, caractéristique du français parlé, met en lumière le caractère extrêmement contraint du codage morphosyntaxique des expressions tropicales en français parlé : il tolère difficilement les arguments topicaux non ratifiés. D'où la formulation par l'auteur du principe de séparation de la référence et de la relation, qui exprime une tendance manifeste à actualiser les topics à fonction argumentale sous forme anaphorique (pronominale ou zéro) et à coder la fonction référentielle du topic en dehors de la proposition. Au sein de cette construction générique, Lambrecht (2002) distingue 7 types de détachements gauches (DG) :

1 - Les DG à SN lié (ex. la vitesse, ça rime plus à rien).

2 - Les DG à SN lié à un argument zéro (ex. ça j’aime pas).

3 - Les DG à SP lié (ex. avec Michel, hier, on est allé se promener à Passy).

4 - Les DG à SN non lié (ex. la mer, tu vois de l'eau).

5 - Les DG à topic en chaîne (ex. tu sais, ma fille Laure, son copain, il est allemand).

6 - Les DG à topic inactif (ex. et les voitures, quand j'ai fait mon apprentissage en Lorraine, on avait des voitures).

7 - Les DG “Mad Magazine” (Lambrecht 1990, ex. Jean, docteur!, je n'en crois pas mes yeux).

\subsection{Les marqueurs syntaxiques du détachement to- pical}

Pour notre part, nous distinguons 5 types d'articulation syntaxique entre un constituant extrapropositionnel thématique (CET) et une proposition se dégagent de notre corpus. Ils mettent en cause la structure syntaxique du CET (cf. 
type II) et le type de rattachement de la proposition au CET ou exceptionnellement du CET à la proposition (cf. ex. 12).

(I) Le premier type est celui d'un énoncé composé d'un CET et d'une proposition consécutive tels que le CET est le support anaphorique du pronom clitique sujet de la proposition, ex.

(1) scur Thérèse, elle surfe très bien sur le web

(2) alors le spot, il se passe comme ça

(3) Dominique, elle est plus habituée

(4) la langue française, elle est compliquée mais elle est drôle

(5) la construction verbe + adjectif invariée, elle ne figure pas dans cette typologie

et avec une reprise pronominale disjointe du CET :

(6) alors cet autre film, alors lui, il est encore plus simple que cette histoire

Pour les occurrences ci-dessus à thème non animé, le pronom clitique non neutre $i l(s)$, elle(s) est remplaçable par le pronom clitique neutre $c ̧ a / c$ ' :

(2') alors le spot, ça se passe comme ça

(4') la langue française, c'est compliqué mais c'est drôle

(5') la construction verbe + adjectif invariée, ça ne figure pas dans cette typologie

Cependant la construction en $\left\{\right.$ Thème $\left._{<\text {-animé> }}, c ̧ a \mathrm{~V}\right\}$ peut présenter une nuance d'ordre méro- ou métonymique (dans le spot, ça se passe comme ça; se débrouiller avec la langue française, c'est compliqué mais c'est drôle). Inversement les occurrences qui suivent présentent une reprise $\mathrm{du}$ thème par ça auquel on peut difficilement (6-8) voire pas du tout (9-10) substituer un pronom non neutre : 
(6) la boulimie, ça coupe des autres

vs. ?elle coupe des autres

(7) une voix, ça se cultive aussi

vs. ?elle se cultive aussi

(8) le babil, c'est très expressif

$v s$. ?il est très expressif

(9) mon mot préféré, c'est aujourd'hui

vs. *il est aujourd'hui

(10) le premier exemple que vous donnez, c'est Oasis vs. * il est Oasis

Un cas particulier est à mentionner, celui où un CET collectif est repris par un pronom clitique pluriel. Ainsi en (11) le Splendide est un collectif d'artistes, qui peut constituer le support anaphorique d'un pronom pluriel.

(11) Alors le Splendide, ils m'ont embauchée

(II) Le second type est celui d'une succession de deux propositions telles que la première est de nature existentielle, typiquement : il y a un $\mathrm{N}$, et que la seconde a pour sujet un pronom clitique sujet coréférent avec le $\mathrm{N}$ dont l'existence est déclarée dans la première, ex.

(12) [il y avait un homme] proposition existentielle support d'un thème indéfini [il voulait absolument être majorette] proposition rattachée

La proposition existentielle n'est pas auto-suffisante et en ce sens la seconde est équivalente à une relative déterminative :

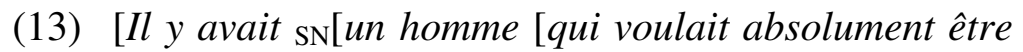
majorette $\left.]]_{\mathrm{SN}}\right]$

La différence pragmatique entre (12) et (13) est que la construction relative déterminative ne permet pas à l'anté- 
cédent un homme d'avoir un statut rhématique autonome : il est dit d'un homme qui voulait absolument être majorette qu'il est présent dans l'univers du discours. En revanche, en (12) il est dit d'un homme non spécifié qu'il a à voir avec l'univers du discours. Ce qu'il a à voir avec cet univers est énoncé séparément par la seconde proposition. La proposition existentielle (rhématique) permet alors à un constituant indéfini (cf. *un homme, $\left[\begin{array}{ll}\text { il ... }\end{array}\right]_{\mathrm{P}}$ ) mais non générique (cf. un homme, $[c ̧ a \ldots]_{\mathrm{P}}$ ) de fonctionner comme un thème pour le co-texte postérieur sans être lié au cotexte antérieur.

(III) Un troisième type est constitué d'un CET et d'une proposition consécutive tels que le CET est le support anaphorique d'un pronom clitique occupant une fonction d'objet direct ou indirect (cf. 14, 15) ${ }^{4}$ :

(14) Marie, tu ne l'as pas revue ces jours derniers?

(15) $\bullet$ Thomas, tu lui as rappelé notre rendez-vous de demain?

Cette configuration est à distinguer clairement de deux cas où une proposition est précédée d'un syntagme prépositionnel extrapropositionnel. Dans le premier cas, nous avons réellement affaire à une opération de détachement à gauche à partir de la position [t] symbolisant la trace et l'origine du mouvement, le constituant prépositionnel étant un actant (objet indirect) antéposé, ex.

(16) $\bullet$ A Thomas $\boldsymbol{t}_{\mathbf{t}}$ j'ai bien rappelé $[\mathbf{t}$ ] notre rendez-vous de demain, mais à sa soeur je ne me souviens plus du tout.

4 Les exemples (14, 15 et 16) sont forgés en l'absence de configurations de ce type dans notre corpus. Le symbole $\bullet$ sera dorénavant utilisé pour marquer ce type d'exemples. 
Tout à fait différent est le cas du positionnement en tête d'un constituant prépositionnel à fonction circonstancielle. Ce type de constituant extrapropositionnel n'est pas thématique, mais correspond à la fonction de cadrage (setting) évoquée par Dik (1997: 396-8). Ainsi en (17), moi est bien un CET, mais avec l'anglais est un constituant de cadrage :

(17) moi, avec l'anglais, j'ai des p'tits problèmes thème constituant de cadrage proposition rattachée

(III') Une variante du troisième type est représentée par le cas (en progression rapide en français oral actuel) où le pronom clitique objet n'est pas mentionné. Le CET constitue alors le support d'une relation anaphorique non instanciée :

(18) $\bullet$ Un voyage aux Caraïbes en avril, je [Ø] prends vs. Un voyage aux Caraïbes en avril, j'en prends un

(19) Les ONG donneuses de leçon, je ne [Ø] donne plus ! vs. Les ONG donneuses de leçon, je ne leur donne plus rien

(IV) Le quatrième type est celui des énoncés à CET faiblement lié à la proposition consécutive. Le lien entre le CET et la proposition consécutive est accessible sans prendre en compte le co-texte antérieur - contrairement au type (v) - mais il passe par l'identification d'une opération de métonymie, ex.

(20) le pain fantaisie, le prix était libre

(21) l'Académie française, je me vois mal dans cet emploi

En (20) le prix est lié à le pain fantaisie par une relation de contiguiité conceptuelle (propriété définitoire de la métonymie) : tout pain a nécessairement un prix, les pains conventionnés ayant un prix fixe et le pain fantaisie un prix libre. En (21) l'Académie française n'est pas un 'emploi', 
mais en être membre constitue une fonction culturelle et sociale assimilable à la rigueur à un emploi. Le déterminant anaphorique cet ne peut donc renvoyer qu'au statut de membre de l'Académie.

(IV') Certaines configurations avec reprise du thème par le pronom neutre ça constituent une variante du type (iv), ex :

(22) la plante, c'est très compliqué

(23) l'Académie Goncourt, c'est beaucoup plus agréable

(24) tous les sports, $c$ 'est que des mots anglais

(25) la règle de participe-passé, c'est extrêmement difficile

(26) la dictée, c'est venu de Lire Magazine

Ce qui est très compliqué c'est l'orthographe des plantes, (c'est Bernard Pivot qui parle), ce qui est beaucoup plus agréable (par rapport à l'Académie française), c'est de faire partie de l'Académie Goncourt, c'est le vocabulaire des sports qui est constitué de mots anglais, ce qui est extrêmement difficile c'est de manier la règle du participe passé (vs. la règle du participe passé, elle est très compliquée), enfin ce qui est venu de Lire Magazine, c'est l'idée de soumettre des candidats à une dictée dans les médias écrits ou télévisuels. Dans aucun de ces cas (22-26) on ne peut à proprement parler identifier une ellipse, mais on peut être sûr qu'il manque un lien conceptuel dans le rattachement de la proposition au CET.

(V) Enfin le cinquième type est celui des énoncés à CET sans lien immédiatement accessible avec la proposition consécutive, ex. $(27,28)$ :

(27) Loc1 : Papa, et la kangoo, elle marche?

Loc2 : Ah la Kangoo, j'crains l'pire 
(28) Loc1 : on aimerait vous voir membre de l'Académie Goncourt

Loc2 : l'Académie Goncourt, j'aimerais bien

En présence d'un énoncé à thème sans lien avec la proposition qui suit, l'allocutaire est obligé de se représenter un contenu propositionnel complet comprenant une référence au CET, en général à partir du co-texte antérieur. Ainsi en (27) la crainte du Loc2 concernant la Kangoo est relative à la capacité de la voiture évoquée à satisfaire la déclaration " elle marche". C'est à propos de la disponibilité immédiate de la Kangoo comme véhicule que le Loc2 craint le pire. En (28), le locuteur aimerait bien être admis dans le cénacle de l'Académie Goncourt et l'allocutaire doit reconstituer le contenu prédicatif entre crochets $(28 ')$ :

(28') l'Académie Goncourt, j'aimerais bien <en faire partie>

\subsection{Les ressources intonatives}

Le modèle que nous proposons pour aborder la structure prosodique des énoncés oraux, s'inscrit dans les approches morphologiques hiérarchiques de l'intonation. Un tel traitement suppose de voir le continuum prosodique comme une entité structurée autour de points intonatifs clés, descriptibles au plan formel en termes de contours et interprétables sous l'angle fonctionnel comme des morphèmes intonatifs, ou intonèmes, c'est-à-dire des objets qui se laissent décrire à la fois par leurs caractéristiques objectales signifiantes et leur contenu interprétatif. L'approche est hiérarchique, dans la mesure où l'intonation est supposée recevoir des niveaux de traitement sémantico-pragmatique l'information nécessaire à son interprétation. En ce 
sens, nous envisageons une primauté ontogénétique du module sémantico-pragmatique dans la dérivation des structures intonative et syntaxique du message. Ces dernières coopèrent par le biais de rendez-vous structurels nombreux pour, non seulement segmenter l'énoncé en segments thématiques et rhématiques, mais également indiquer la structure interne de ces derniers (pour un point de vue identique, voir Morel \& Danon-Boileau 1998). De ce cadre théorique découle notre stratégie de traitement : pour mettre au jour le rôle fonctionnel de l'intonation, en particulier comme indicateur de détachement, nous nous fondons sur une méthode inductive qui se décompose en deux volets, appelés respectivement concret, et représentationnel. Le premier concerne la matérialité des faits prosodiques tels qu'ils sont observés dans le signal de parole, le second est dédié à la représentation phonologique formelle de ces observables. Ce second niveau constitue une médiation nécessaire pour rendre compte ensuite de la fonctionnalité des objets prosodiques, à savoir: les fonctions communicatives qu'ils servent dans le langage. C'est à ce stade du traitement que sont identifiés les différentes relations prosodiques que peuvent contracter les groupes intonatifs identifiés dans la chaîne parlée. Pour préciser la spécificité de notre approche par rapport aux modèles morphologiques standards, deux points doivent être soulignés : (i) le refus du primat de la syntaxe pour la dérivation des structures (figures 1.a, 1.b), (ii) le caractère ascendant du traitement : les morphèmes intonatifs ne constituent pas des primitives données en entrée mais émergent par induction à partir du repérage phonétique de contours mélodiques (figure 2). 


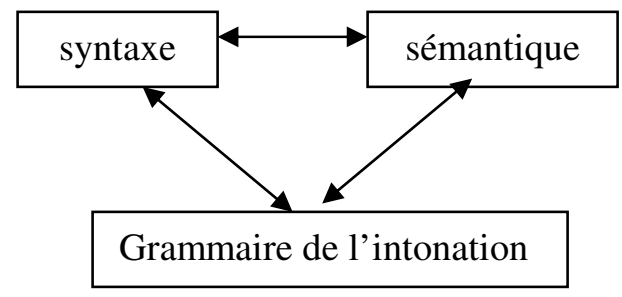

Figure 1.a. Hiérarchie des modules d'après Rossi (1999: 52)

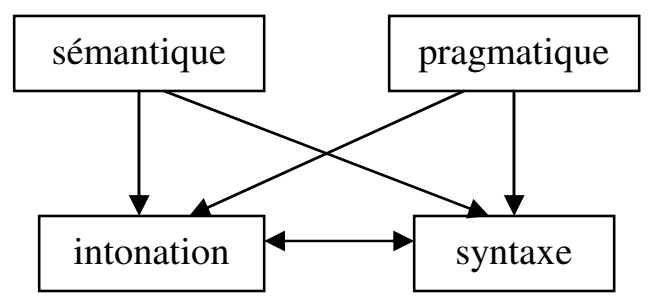

Figure 1.b. Hiérarchie des modules d'après Lacheret (2002) 


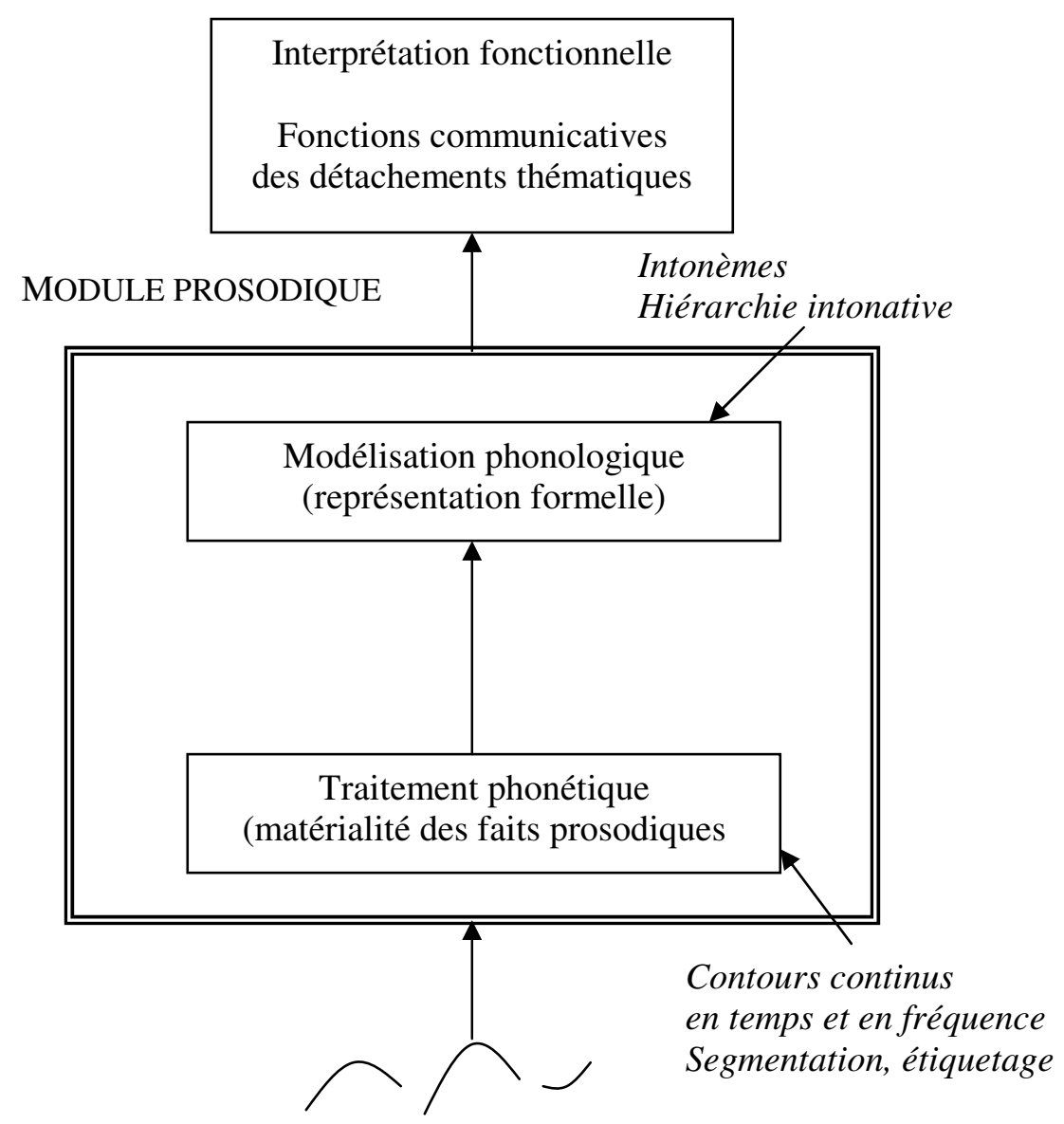


Figure 2. Traitement inductif : de l'observation des données prosodiques à l'interprétation fonctionnelle

- Matérialité des faits prosodiques

Le niveau ici décrit concerne le traitement phonétique des données sonores, c'est-à-dire l'étude des paramètres physiques mobilisés pour actualiser le système intonatif dans la substance. La question est la suivante : comment, malgré son extrême hétérogénéité, transformer de la matière brute en données manipulables et interprétables pour l'expérimentateur? Les problèmes de segmentation et d'étiquetage des unités à manipuler s'avèrent donc centraux. Nous situant dans une approche globale de l'intonation ${ }^{5}$, nous proposons une description du continuum prosodique en termes de gestes, ou contours, continus en temps et en fréquence. Toute variation significative de la fréquence fondamentale et/ou de la durée, calculée par rapport à une valeur de base 6 et identifiée en position terminale de mot, est considérée comme la frontière droite d'un groupe intonatif 7 (figure 3 ).

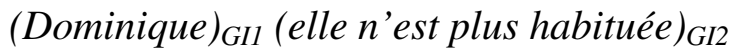

$(\text { La langue française })_{G I 1}$ (elle est compliquée $)_{G I 2}$

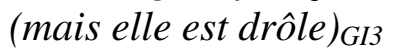

5 Pour la distinction entre modélisation globale et locale de l'intonation, voir Lacheret \& Beaugendre (1999: 100).

6 Pour la fréquence fondamentale, la valeur de base dépend du registre mélodique moyen du locuteur, pour la durée, elle correspond à la durée moyenne des syllabes non terminales de mots.

7 Il s'agit là d'un traitement acoustique effectué à l'aide du logiciel winpitch (Léon \& Martin 2000). 


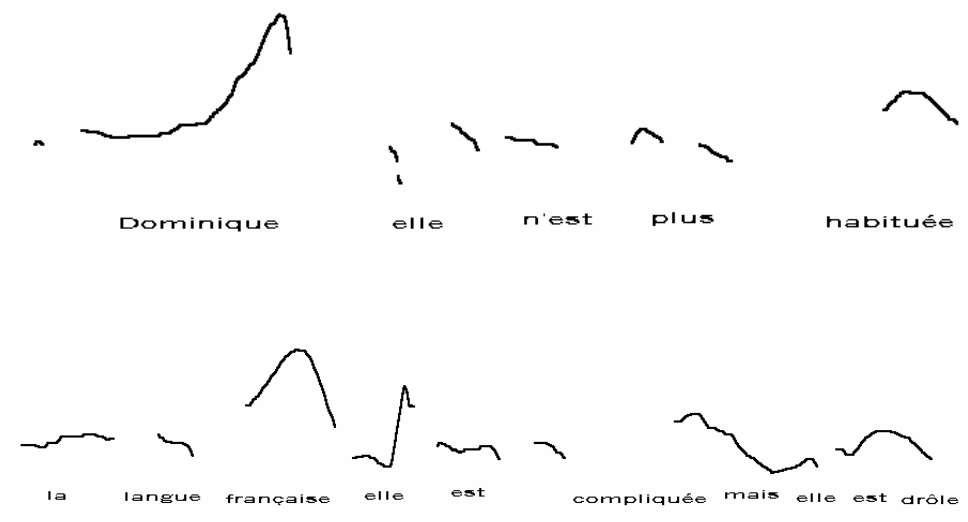

Figure 3. L'actualisation des groupes intonatifs dans la parole

Notre stratégie d'analyse est ainsi axée sur la modélisation des proéminences accentuelles terminales de mots, considérées comme des marqueurs perceptifs que l'auditeur utilise pour segmenter le continuum sonore en groupes intonatifs successifs. Ces proéminences sont décrites par un faisceau de traits, représentant les propriétés phonétiques nécessaires et suffisantes pour les caractériser formellement et les interpréter fonctionnellement ensuite (figure 4). Les informations fournies par la matrice de traits sont fondées sur un principe de valeur relative, selon lequel la valeur fonctionnelle des unités se définit à la fois à partir du repérage d'oppositions paradigmatiques 8 et de l'enchaînement syntagmatique des contours porteurs des proéminences et de leur contexte immédiat. En d'autres termes, la valeur

8 Un contour prend sa valeur relativement à d'autres qui lui sont substituables et forment son paradigme de définition. 
d'un contour dépend non seulement de la valeur de ceux avec lesquels il pourrait se substituer dans un contexte terminal de mot, mais également de la configuration du contour terminal du groupe qui le précède et également de son entourage proche. Ainsi, la présence éventuelle d'une pause subséquente à un contour peut modifier la valeur de ce dernier et prendre elle-même différentes valeurs relativement à son environnement. L'interprétation fonctionnelle qu'on peut en faire (conclusive ou continuative) dépend, en effet, de la présence éventuelle d'un euh d'hésitation antéposé ou postposé. Quatre types d'informations sont donc codés : le profil d'un contour terminal de groupe intonatif, le niveau fréquentiel qu'il traverse, son caractère allongé ou non et son contexte droit (présence éventuelle d'une pause et/ou d'un euh d'hésitation).

$$
\left\{\begin{array}{l}
\mathrm{T} \\
\mathrm{M} \\
\mathrm{D} \\
\mathrm{MD}
\end{array}\right\}\left\{\begin{array}{l}
\mathrm{SA} \\
\mathrm{A} \\
\mathrm{M} \\
\mathrm{G} \\
\mathrm{IG}
\end{array}\right\}\left\{\mathrm{l}^{\prime}\right\}\{\mathrm{H}\} \quad\{\#\}\{\mathrm{H}\}
$$

Figure 4. Matrice de traits associés aux contours terminaux

où, de gauche à droite, sont indiqués : (i) les caractéristiques prosodiques d'un contour relatives à sa direction (montant, 'M', descendant, 'D', dynamique, 'MD' ou statique 'T'), le niveau traversé par un contour (de suraigu à infragrave) et son caractère allongé ou non (marqueur ' :') ; (ii) l'environnement droit du contour, le cas échéant : euh préposé à une pause (Hésitation), pause (silence ou inspiration), euh postposé (H) 


\section{- Représentation formelle}

Si les proéminences accentuelles terminales de mots constituent d'abord des indices perceptifs utilisés par l'auditeur pour segmenter le continuum sonore en groupes intonatifs successifs, elles lui permettent également d'estimer les relations d'inclusion ou, au contraire, d'autonomie qui unissent les groupes en présence, cela indépendamment des contraintes syntaxiques qui pourraient les sous-tendre. En conséquence, le module phonologique ici décrit a pour rôle de fournir une représentation de la hiérarchie intonative sous-jacente à l'actualisation des groupes intonatifs émergents à l'issue du traitement phonétique. C'est autour des traits utilisés pour coder ces catégories que s'effectue le passage entre les deux modules. Dans un premier temps, il s'agit de statuer sur le potentiel d'intégration intonative d'une unité thématique. La règle est la suivante: tout segment thématique non accentué est intonativement intégré à la chaîne rhématique qui le suit $\left.(\text { ex. ça, ça n'se voit pas })_{G I}\right)$. En fait, ce type de structure est pour l'heure inexistant dans nos corpus d'étude. Le thème, en effet, fait toujours l'objet d'une accentuation finale, manifestée par un contour montant dans le niveau aigu par rapport au registre de base du locuteur (cf. supra, figure 3). Pour reprendre la terminologie standard (Rossi \& al 1981), il s'agit là d'un intonème continuatif. La question est alors à nouveau de savoir quelle valeur associer à cet intonème, en termes de ruptures et d'emboîtements potentiels. Il y a détachement maximal lorsque les quatre conditions suivantes sont réunies (voir Lacheret et Victorri 2002 pour une première approche) : 
- présence d'une pause après le continuatif dont la durée dépasse un seuil de $300 \mathrm{~ms}$;

- excursion fréquentielle terminale du contour dans le niveau suraigu ;

- écart mélodique (ou downstep) dépassant un seuil de 3 demi-tons ${ }^{9}$,

- absence d'un euh à proximité de la pause.
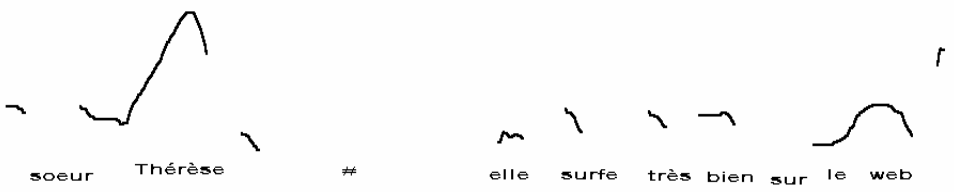

Figure 5. Exemple de détachement thématique pour la séquence sœur Thérèse \# elle surfe très bien sur le web

Plusieurs remarques s'imposent ici. Tout d'abord, les indices de détachement maximal sont à chercher simultanément dans les paramètres activés (durée, fréquence fondamentale) mais également dans les seuils d'activation. Autrement dit, il ne suffit pas qu'il y ait détection d'une pause pour que l'on puisse identifier un détachement, encore faut-il qu'elle soit d'une certaine longueur et accompagnée d'une variation fréquentielle suffisamment marquée. Ensuite, le repérage d'un détachement ne dépend pas de la valeur précise des seuils mais de leur ordre de grandeur. En conséquence, lorsqu'un paramètre est inférieur au seuil mais très proche, le détachement pourra malgré tout

\footnotetext{
9 le donwstep correspond à la différence de hauteur entre le dernier extremum de F0 précédant la pause et la première valeur de f0 suivant la pause.
} 
être identifié si les autres paramètres ont des valeurs nettement au-dessus du seuil. Enfin, l'absence d'un euh contigu à gauche ou à droite de la pause permet d'opposer deux types de pause, l'une structurale, marquant un détachement effectivement planifié par le locuteur pour laisser à son interlocuteur le temps d'intégrer le thème posé avant de prédiquer à son sujet, l'autre, liée au problème de planification du segment rhématique.

Ces deux niveaux de structuration posés (intégration potentielle vs. rupture effective), il s'agit de voir ensuite s'il est possible de faire émerger une granularité plus fine dans les types de relation instanciés. La question est alors la suivante : n'existe-t-il pas des niveaux de détachement et/ou d'intégration intermédiaires ? Autrement dit, outre la manière dont se structurent et s'apparient les objets intonatifs, les uns avec les autres, divers degrés peuvent également être détectés dans les relations établies. Ces dernières sont donc à la fois qualitatives et quantitatives. C'est ici qu'intervient la notion de groupe intonatif affixé (GIA) qui repose sur l'emboîtement de deux groupes intonatifs, l'un étant perceptivement plus saillant que l'autre ${ }^{10}$. Les indices acoustiques utilisés pour calculer cette saillance se fondent sur l'application d'un principe générique de dominance intonative (principe DOM). Selon ce dernier, tout GI domine le GI immédiatement contigu à gauche s'il est marqué soit par une excursion fréquentielle terminale dans le niveau aigu, supérieure d'au moins 1 ton, soit par une

10 Ces phénomènes de saillance ne sont pas spécifiques à la prosodie ni sans doute même au langage, mais correspondent à des fonctions cognitives élémentaires d'organisation de formes, tels qu'on peut les rencontrer en vision par exemple Bonnet 1989). 
descente dans le niveau infra-grave, soit enfin par un contour terminal de pente opposée (figure 6). L'application de DOM donne lieu au regroupement des GI en GIA (intégration de deuxième niveau), dans le cas contraire, la construction intonative révèle un détachement intermédiaire. La structure intonative interne de l'énoncé est ainsi obtenue par le test récursif gauche-droite de ce principe d'inclusion (comparaison de GI1 avec GI2, de GI2 avec GI3, etc.), la présence du trait infra-grave (conclusif majeur) ou d'un continuatif dominant (niveau suraigu) représentant la condition d'arrêt de l'algorithme.

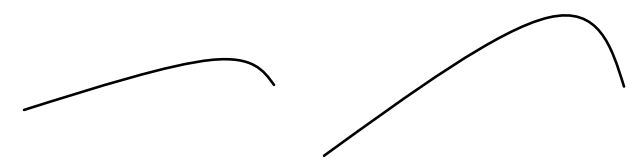

alors le splendide ils m'ont embauchée

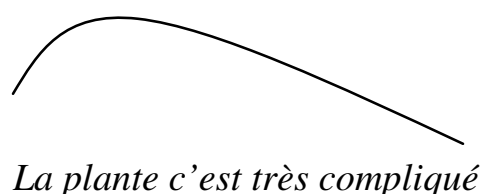

Figure 6. Exemple d'intégration intonative par application du principe DOM

\section{Interprétation fonctionnelle}

L'alignement des patrons intonatifs et syntaxiques rencontrés permet de poser un continuum de détachement topical, interprétable sous l'angle informatif, discursif et cognitif. 


\subsection{Alignement inotonsyntaxique}

Les différentes configurations prosodiques, considérées indépendamment de la syntaxe, mettent en lumière plusieurs degrés de saillance thématique. Un thème est phonétiquement peu saillant lorsqu'il entretient une relation d'emboîtement intonatif avec le segment rhématique qui le suit, la rupture intonative de niveau 1 indique une amorce de détachement, la rupture de niveau 2 constitue un marqueur de détachement explicite (figure 7).

\begin{tabular}{cccc}
- & Saillance thématique & + \\
\hline GIL1 & GIL2 & GID1 & GID2
\end{tabular}

Figure 7. Les différents degrés de détachements intonatifs comme indices de saillance thématique où 'GIL' = groupe intonatif lié, 'GID' = griype intonatif détaché

Ainsi, pour une même structure syntaxique à détachement gauche, SN[PRO-V-(SX)] par exemple, il sera possible de distinguer différents degrés de détachement. Première remarque donc: ce que la syntaxe ne peut pas coder, à savoir un continuum de détachement, la prosodie le marque. Dans certains cas, en revanche, la redondance des marques est fonctionnellement pertinente. Autrement dit, un thème qui fait l'objet à la fois d'un détachement prosodique fort et d'un déploiement syntagmatique (entité introduite par une proposition existentielle, ou insérée dans une chaîne thématique) sera particulièrement saillant (cf. exemple 12 en supra). En conséquence, pour l'observateur, la combinaison des indices syntagmatiques et intonatifs permet de préciser ce continuum de détachement théma- 
tique en associant à chaque type de CET un poids, d'autant plus fort que le détachement prosodique est important (soit 4 poids intonatifs). Pour le marquage syntaxique, on oppose le thème extrait accompagné d'un déploiement syntagmatique ( $\mathrm{DS}+$, poids 2 ), au thème extrait codé par un et un seul syntagme, voire une seule unité lexicale (DS-, poids 1).

\begin{tabular}{|c|c|c|c||c|c|c|}
\hline GIL1 & GIL2 & GIR1 & GIR2 & DS+ & DS- & $\begin{array}{l}\text { Poids de } \\
\text { saillance }\end{array}$ \\
\hline \hline- & - & 1 & - & - & 1 & 2 \\
\hline- & - & - & 2 & - & 1 & 3 \\
\hline- & - & 1 & - & 2 & - & 3 \\
\hline- & - & - & - & 2 & - & 4 \\
\hline 0 & - & - & - & - & 1 & 1 \\
\hline- & 0,5 & - & - & - & 1 & 1,5 \\
\hline
\end{tabular}

Nous souhaitons commenter ce tableau par trois points :

1. Etant donné ce qui a été dit en supra, sur l'inexistence dans nos corpus de thème inaccentué, le poids 1 n'est là qu'à titre prospectif (dans l'attente d'une occurrence de ce type).

2. L'intégration perceptive du détachement résulte de deux niveaux de traitement en parallèle : syntaxique et prosodique.

3. Les contraintes structurales, de nature syntagmatique, bloquent certaines configurations prosodiques. Ainsi, un thème qui se déploie sur l'axe syntagmatique (DS+) ne pourra jamais être intégré prosodiquement. De prime abord donc, il y a bien une dépendance explicite de la prosodie vis à vis de la syntaxe. En fait, il s'agit là d'une contrainte interne purement rythmique: la longueur du syntagme 
thématique est, bien entendu, associée à la production d'une chaine syllabique trop longue pour faire l'objet d'une désaccentuation. Ensuite, le caractère dominant du contour marquant la fin du topic, provenant d'une instruction pragmatique spécifique, bloque toute possibilité d'emboîtement : l'intonème qui le marque, dominant dans la structure intonative globale (voir aussi Rossi 1999), signale de façon iconique que l'attention de l'interlocuteur doit se porter en priorité sur le constituant en question.

\subsection{Accessibilité cognitive, stratégies illocutoires et enjeux co-énonciatifs}

Selon l'approche informative adoptée par les grammaires fonctionnelles, le codage du topic est étroitement lié à son degré d'accessibilité. Ainsi, pour Dik (1989)11, les fonctions pragmatiques spécifient le statut informationnel des constituants à l'intérieur comme à l'extérieur de la prédication, en fonction du cadre communicatif d'ensemble dans lequel ils sont employés. Cette notion de cadre communicatif correspond à l'évaluation de la part du locuteur $\mathrm{du}$ stock d'informations pragmatiques possédé par l'allocutaire au moment du déroulement de l'acte de communication. Une telle conception de l'interlocution conduit l'auteur à opposer différents types de topic. Un topic donné supposera un référent qui est très saillant (donc cognitivement actif) dans la représentation mentale des interlocuteurs. Il correspond à ce sur quoi porte le discours au moment où l'expression ainsi marquée est employée. Les unités codant cette fonction sont la plupart du temps morphologiquement et phonologiquement atténuées -

11 Voir François et Cornish 1995. 
formes $\varnothing$ (ellipses), pronoms clitiques (atones) de troisième personne (la dentiste, elle a dit qu'il fallait que tu prennes rendez-vous). L'instruction ainsi véhiculée à l'interlocuteur consiste à solliciter un effort cognitif faible, le référant en question étant hautement disponible, puisqu'au premier plan de la conscience des uns et des autres. Un sub-topic sert à pointer un référent disponible en puissance par l'intermédiaire d'une référence antérieure, qui va faire l'objet à son tour d'un développement discursif. Les référents auxquels renvoie ce type de topic sont évidemment moins saillants que dans le cas des topics donnés, mais ils sont disponibles, à court terme, au niveau de la mémoire de travail, ils sont donc semi-actifs. Le groupe nominal défini constitue le type d'expression archétypal pour y référer (Justine m'a prêté son cahier pour réviser, mais les pages elles sont toutes tachées). Un topic repris permet de réactiver un topic élaboré bien antérieurement dans le discours mais délaissé par les interlocuteurs depuis lors. Le référent en question est donc inactif au moment où l'expression le ravivant est utilisée. Selon Dik, une telle fonction est codée par un signal de changement topical en même temps que par une expression anaphorique forte ou explicite (nom propre, groupe nominal défini ou démonstratif par exemple). Quelle que soit l'unité employée, il y aura toujours un marqueur pour signaler que le référent pointé fait déjà partie du discours en cours (pour revenir à Charlotte, elle m'inquiète un peu en ce moment). Pour terminer, Dik envisage un topic nouveau, qui correspond à l'introduction d'un référent donné dans un discours au moyen (prototypiquement) d'une expression indéfinie, référent qui est destiné à faire l'objet d'une élaboration dans le discours ultérieur (tiens! j'ai rencontré une des grévistes ce 
matin, tu sais ce qu'elle m'a dit ?). Ce raisonnement est, de notre point de vue, applicable à la prosodie (configuration GIL1 pour un topic donné, GIL2 pour un sub-topic, GID1 pour un topic repris, GID2 pour un topic nouveau). Le critère de disponibilité cognitive (ou saillance mémorielle) est également fondamental chez Lambrecht pour expliquer le principe de séparation du rôle et de la référence, qu'il pose comme premier dans la construction des messages en français parlé et dont la fonction discursive de base est d'introduire un référent topical qui n'a pas été encore ratifié 12 dans le discours. Certes, comme chez Dik, une distinction purement binaire entre unité ratifiée et non ratifiée n'est pas suffisante, d'où l'utilisation par Lambrecht de l'échelle d'accessibilité des référents posée par Chafe (1987) : si un topic est toujours identifiable, il n'est pas nécessairement actif, on a donc bien des degrés d'accessibilité, donc de ratification, qui pourront motiver les différents degrés de détachement intonosyntaxique posés dans notre tableau précédent ${ }^{13}$. Soit le continuum suivant :

12 C'est-à-dire dont le rôle topical à l'intérieur de la proposition n'est pas encore établi.

13 Pour l'heure, nous nous soucions uniquement de l'extraction syntaxique, celle où le topic n'occupe jamais la fonction de sujet argumental. 


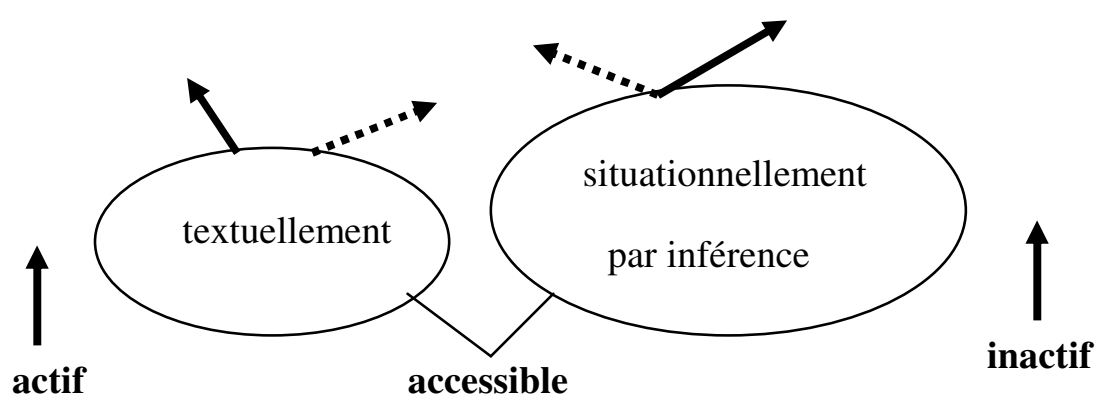

Figure 8. Topic (externe (détaché) ou interne à la clause) : les contraintes d'accessibilité cognitive

Cette approche en terme de saillance mémorielle est certes pertinente, mais elle n'est pas seule à justifier les détachements intonosyntaxiques rencontrés: le locuteur n'est pas une simple machine à produire de l'information, encore le fait-il selon un point de vue spécifique ${ }^{14}$ et avec un certain objectif interlocutoire. Autrement dit, si les différentes opérations de topicalisation, associées au mode de donation du référent, peuvent être expliquées par le statut plus ou moins actif des référents traités, divers facteurs énonciatifs, tels que les aspects interactionnels à l'œuvre dans le dialogue, le contexte discursif, les visées

14 A la fonction représentative se superpose une fonction expressive. 
illocutoire et perlocutoire ${ }^{15}$, fournissent également un cadre interprétatif pour préciser les contraintes qui soustendent l'application du principe de séparation de la référence et de la relation. Ces points nous amènent à compléter notre figure $1 . b$ : les instructions provenant de la structure communicative (module pragmatique) relèvent de deux sous-composantes: une structure informationnelle et une structure discursive indépendante. Cette dernière détermine les mécanismes d'enchaînement discursif, les facteurs de négociation dialogale et les phénomènes de coloration affective manifestés par l'emphase par exemple. Elle n'est, bien évidemment, pas étrangère aux constructions par décondensation caractéristiques de nos corpus d'étude.

Structure informationnelle (thème/focus)

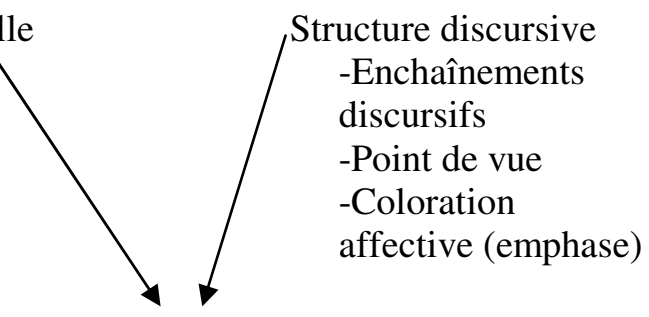

Pragmatique : structure communicative

15 Les messages véhiculent non seulement des informations mais aussi des instructions pour traiter ces informations (Valduvi 1994). 
Figure 9. Les sources pragmatiques des schémas intonosyntaxiques

Au principe de séparation du rôle et de la référence formulé par Lambrecht, on peut superposer le principe de monstration. L'essentiel, en effet, pour le locuteur, qui construit l'énoncé par touches successives “ n'est pas tant d'insister sur ce qu'il dit de nouveau que de bien montrer le point d'où il part" (Morel \& Danon-Boileau 1998: 37). Cet angle d'attaque se superpose donc aux approches classiques sur le statut des référents, qu'elles soient formulées en termes textuels ou cognitifs (notion d'identifiabilité du référent et de saillance mémorielle). Il fait appel à une autre notion, fondamentale pour notre système de représentation : celle de scène verbale :

Au cours de l'activité de parole, se produit un processus cognitif très particulier, qui consiste pour le locuteur à essayer de construire un espace extérieur à lui-même, partageable par ses interlocuteurs, et dans lequel il donne à voir ce dont il parle comme une scène projetée devant eux. L'acte d'énonciation de base serait donc constitué d'au moins deux éléments : la description d'une scène et la donnée d'un point de vue sur cette scène, ce deuxième élément étant essentiel pour permettre le partage de la "vision". Victorri \& Fuchs (1996 : 200).

Ainsi, la saillance et l'arrière plan sont à situer également et surtout sur la scène verbale partagée par les interlocuteurs, et la place d'une entité sur la scène n'est pas toujours dépendante du fait qu'elle soit nouvelle ou déjà connue, ou encore saillante dans la mémoire des interlocuteurs. Sous l'angle de la visée illocutoire donc, les variations de saillance sont conditionnées par l'engagement de 
l'énonciateur dans son discours et par l'effet qu'il veut produire sur son co-énonciateur (voir également Morel \& Danon-Boileau 1998). Ce point est d'ailleurs implicitement reconnu par Dik qui ne réduit pas la notion de stock d'informations pragmatiques à l'ensemble des données connues par l'allocutaire, ou supposées comme telles par le locuteur, mais qui y inclut également les croyances, sentiments et idées préconçues du co-énonciateur. Quand Dik affirme que la dimension topicale reflète l'évaluation que fait le locuteur du stock d'informations pragmatiques de son interlocuteur à tout moment du déroulement de l'acte d'énonciation, il dit également qu'en fonction de cette évaluation, l'objectif du locuteur est d'amener ce dernier à modifier d'une certaine manière ce stock, soit en y introduisant de nouvelles entités ou de nouvelles propriétés ou relations attribuées à des entités déjà en place, soit en éliminant du stock en question des entités ou propriétés données 16 . Autrement dit, produire un discours, c'est agir sur l'autre et, le cas échéant, se positionner par rapport à ses croyances, voire les réorienter. Pour ce faire, les stratégies mises en œuvre dans l'articulation discursive sont fondamentales. A ce titre, les constituants extra-propositionnels, détachés à gauche permettent non seulement d'ancrer la prédication à venir, mais également de gérer l'interaction verbale dans son ensemble et d'organiser ce contenu par rapport au contexte discursif global. Il est donc nécessaire d'appréhender la notion de détachement dans un

16 A l'inverse des constituants détachés, des objets de discours qui ne sont plus relancés par l'intermédiaire de marqueurs linguistiques spécifiques auront tendance à disparaître d'eux-mêmes et à quitter la scène. 
cadre discursif plus large que celui constitué par le segment $<$ thème + prédicat $>$. Les constituants thématiques détachés en position frontale servent ainsi souvent à assurer la transition entre un thème développé dans une unité discursive précédente et celui d'une nouvelle unité qu'ils servent à délimiter. Au sein de ce cadre discursif global, l'autonomie intonosyntaxique du thème est nécessaire pour, dans bien des cas, lui conférer son propre statut illocutoire (cf. supra 2.1 : l'analyse de Dick). L'instanciation du segment thématique correspond à la production d'un acte spécifique qui le plus souvent constitue une reprise en écho d'un segment antérieur dans le discours. Il est notamment très productif dans les situations d'interaction dialogale (où tu as mis le journal ? le journal, ben il est sur la table), Ce marqueur de force illocutoire indique en quelque sorte la direction d'ajustement entre le contenu propositionnel et la référence. L'intonation est, là encore, essentielle puisqu'elle permet de distinguer les deux fonctions que peut jouer le CET (mode de donation d'un topic ou reprise en écho). Pour conclure :

ce constituant de tête peut jouer deux rôles qui s'excluent mutuellement. Il peut jouer le rôle de déictique 17 , le locuteur montre ce dont il va parler (...) dans ce cas nous dirons que $\mathrm{T}$ est chargé d'une valeur contextuelle référentielle, car l'allocutaire prend connaissance du référent avant de recevoir l'information qui le concerne. Dans une seconde hypothèse, le locuteur répond à une question explicite ou implicite de l'allocutaire (...) Dans ce cas, le constituant de tête, écho de la question, coïncide avec le donné et il acquiert une valeur contextuelle inférentielle, puisque de la réponse on peut inférer qu'une

17 Voir en supra, la présentation du principe de monstration. 
question a été posée ou que simplement l'allocutaire attend une information à son sujet précis (Rossi 1999 : 65).

Ces différences sont marquées intonativement: un thème repris en écho est nécessairement marqué par un geste terminal dans le niveau suraigu et ponctué d'une pause, la marge de manœuvre étant plus souple pour le thème à valeur déictique.

\section{Conclusion}

L'angle d'attaque qui a été le nôtre pour aborder la notion de détachement thématique s'inscrit dans une perspective psycholinguistique, dans laquelle la notion de représentation mentale est centrale. L'activité du langage est vue comme la construction d'une représentation d'une certaine réalité et selon un certain éclairage, que l'on a désignée par le terme de scène verbale et qu'on pourrait nommer, à l'instar de Grize (1990), schématisation discursive. $\mathrm{Si}$ le terme est d'abord appréhendé dans une perspective dynamique et entendu au sens de processus (construction de scène), il correspond également à un résultat (la représentation qui en dérive). Ces deux phases convoquent et le locuteur et l'allocutaire. En conséquence, nous avons voulu prendre en compte à la fois les mécanismes de production et les traitements perceptifs qu'ils induisent, les premiers étant révélateurs des intentions communicatives du locuteur, les seconds de son exploitation par l'interlocuteur. Nous avons souhaité montrer que derrière le principe de séparation du rôle et de la référence se cache un principe beaucoup plus fondamental encore pour la construction de la scène : le principe d'ancrage, qui se matérialise dans le discours par un principe de quan- 
tité. Pour préciser ces notions d'ancrage et de quantité : les constituants extrapropositionnels thématiques, qui ne sont ni des propositions, ni constitutifs d'une proposition, assurent clairement une fonction de signalisation interpropositionnelle spécifique, radicalement différente de celle des connecteurs par exemple. Tandis que les seconds relient deux constituants à fonction prédicative, les premiers établissent le lien entre fonction référentielle et fonction prédicative, assurant ainsi à la proposition consécutive un fondement référentiel (extrapropositionnel) qui lui permet d'exercer pertinemment sa fonction prédicative. Le principe de quantité, quant à lui, est d'abord associé à la redondance des marques et permet d'isoler une figure thématique dans un fond discursif. Il explique non seulement la mobilisation conjointe des niveaux de traitement intonatif et syntaxique mais également les déploiements syntagmatiques éventuels sur le segment thématique et/ou le nombre de traits prosodiques et les seuils activés pour produire une figure thématique, la configuration \{+ample, +aigu, +pause \} marquant l'application phonétique prototypique d'un tel principe (voir l'hypothèse de l'effort articulatoire chez Gussenhoven 2002). Nous avons voulu montrer ensuite que les différents degrés de détachement intonatif mobilisés pour actualiser une figure thématique sont à corréler simultanément avec des critères informatifs et discursifs. Sous l'angle informationnel, il s'agit de régler les problèmes de l'accessibilité référentielle. Du point de vue discursif, les détachements variables sont liés aux enjeux co-énonciatifs (correction d'une présupposition pragmatique ou d'une croyance erronée, négociation de point de vue par exemple) et aux problèmes de cohésion textuelle. Dans tous les cas, les différents schémas théma- 
tiques rencontrés répondent au principe général de pertinence, selon lequel un énoncé est d'autant plus pertinent que le coût cognitif pour le comprendre est faible : l'activation d'unités thématiques saillantes permet une coconstruction de la scène verbale plus immédiate, cette dernière subissant au cours de l'échange verbal des transformations, jusqu'à un état final stabilisé car partagé par les interlocuteurs. A partir de ce stade, les objets de discours n'ont plus à être relancés et les détachements thématiques se font de plus en plus ténus jusqu'à disparaître complètement.

Un tel angle d'attaque pourrait être avantageusement enrichi par une prise en compte des facteurs relatifs aux problèmes de planification du message (mémoire et économie cognitive, ordre de stockage des informations, processus d'encodage, récupération de l'information en mémoire, etc.). Cela suppose que les expérimentations effectuées sur l'écrit puissent être transposées judicieusement sur l'oral (voir. Olive 2002 pour un bilan des différentes techniques d'expérimentation). 


\section{Bibliographie}

Apothéloz D. 1997, “Les dislocations à gauche et à droite", in Miéville \& Berrendonner (éd.), 183-218.

Baddeley Alan D. 2000, “The episodic buffer: A new component of working memory?", Trends in Cognitive Sciences, 4, 417423.

Bonnet $\mathrm{Cl}$. 1989, "la perception visuelle des formes", Traité de psychologie cgnitive, Paris, Dunod, 3-82.

Chafe W. 1987, "Cognitive Constraints on Information Flow", in Coherence and Grounding in Discourse, R. Tomlin (éd.), Amsterdam, Benjamins, 21-51.

Dik S. 1989,

Dik S.

1997, The Theory of Functional Grammaar, Part 2 : Complex and derived constructions, Beflin, De Gruyter.

Fayol M. 2002 éd, Production du langage, Paris, Lavoisier.

Ferrand L. 2002, " les modèles de production de la parole ", in Michel Fayol (éd.), 27-44.

François J., Cornish F. 1995, “Le modèle néerlandais de grammaire fonctionnelle", l'information grammaticale, 67, 12-20.

Grice H. 1975, "Logic and Conversation", in Syntax and semantics 3 : Speech Acts, P. Cole \& J.L. Morgan (éd.), New York, Academic Press, 41-58.

Grize J.B. 1990, Logique et langage, Paris, Ophrys ?

Guillaume P. 1937, La Psychologie de la forme, Paris, Flammarion.

Guimbretière E. 2000, La prosodie au cour du débat, Rouen, Dyalang. 
Gussenhoven C. 2002, "Intonation and Intepretation ", Speech Prosody, Aix-en-Provence, D. Hirst (éd.), 47-57.

Hagège $\mathrm{Cl}$. 1978, " $\quad$ Du thème au thème en passant par le sujet. Pour une théorie cyclique", La linguistique, 14/2, 2-38.

Halliday M.A.K. $\quad 1985$, An Introduction to Functional

Halliday M.A.K, Hasan R. Grammar, London, Arnold.

1976, Cohesion in English, London, Longman, English Language Serie 9.

Lacheret A., Beaugendre F. 1999, la prosodie du français, Paris, CNRS.

Lacheret A. 2002, Modélisation prosodique $d u$ français parlé, diplôme d'habilitation à diriger des recherches, Paris $\mathrm{X}$.

Lacheret A. 2003 à paraître, La prosodie des cir-

Lacheret A, Victorri B. constants, Louvain, Peeters.

2002, "La période intonative comme unité d'analyse pour l'étude du français parlé : modélisation prosodique et enjeux linguistiques ", Verbum, XXIV, 5572.

Lambrecht K. 1990, "What, me worry? Mad Magazine sentences revisited", in BSL 16, 215-228.

Lambrecht K. 1994, Information Structure and Sentence Form, Cambridge University Press.

Lambrecht K. 1998, "Sur la relation formelle et fonctionnelle entre topics et vocatifs", Langues, 34-45.

Lambrecht K. 2002, "Contraintes cognitives sur la structure de la phrase en français parlé ", Conférence Lattice, Université de Paris 3, juin 2002. 
Leon P., Martin Ph. 2000, “Prosodie et technologie ”, in E. Guimbretière (2000 éd.), 135-150.

Levelt Willem J.M. 1989, Speaking from intention to arti-

LI Ch., Thompson S. culation, Cambridge, GB. Cambridge.

1976, "Subject and Topic: a new typology of language ", in Li (éd.), Subject and Topic, New York, Academic Press, 457-489.

Miéville D., Berrendonner A.

1997, Logique, discours et pensée, Bern, Peter Lang.

Morel M.A., Danon-Boileau L.

1998, Grammaire de l'intonation, l'exemple du français, Paris, Ophrys.

Olive T. 2002, "La gestion en temps réel de la production verbale: méthodes et données ", in Michel Fayol (éd.), 131-147.

Rossi M. \& al. $\quad$ 1981, L'intonation, de l'acoustique à la sémantique, Paris, Klincksieck.

Rossi M. 1999, L'intonation, le système du français, Paris, Ophrys.

Roulet E., Filliettaz L., Grobet A.

2001, Un modèle et un instrument d'analyse $d u$ discours, Bern, Peter Lang.

Simon A.C.

2002, Segmentation et structuration prosodique du discours, thèse de doctorat, université de Louvain.

Valduvi E. 1994, "The Dynamic of Information Packaging ", in Integrating Information Structure into Constraints-based and Categorial Approches, Yana-2 Report R1.3.B, ILLC, Amstedam, E. Engdahl (éd.), 1-26.

van Valin Jr. R.D. $\quad$ 2001, An Introduction to Syntax, Cambridge (GB), Cambridge University Press.

van Valin Jr. R.D. \& R. LaPolla 
1997, Syntax : Structure, Meaning, and Function, Cambridge (GB), Cambridge University Press.

van Valin Jr, R.D. (à paraître), The Syntax-Semantics-Pragmatics Interface: An Introduction to Role and Reference Grammar, Cambridge (GB), Cambridge University Press.

Victorri B., Fuchs C. 1996, La polysémie. Construction dynamique du sens, Paris, Hermès. 